Zukunftswerkstatt

Open Access

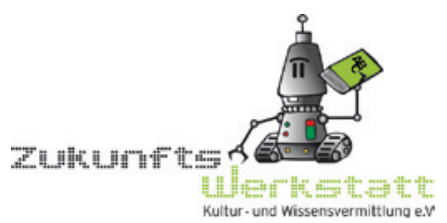

\title{
Das „Internet der Dinge“ - wenn Alltagsgegenstände sich vernetzen
}

DOI 10.1515/bd-2014-0101

Das „Internet der Dinge“ („Internet of things“, „IoT“), bei dem Maschinen, Geräte und Sensoren autark über das Netz miteinander kommunizieren, ohne dass Menschen eingreifen („Maschine-zu-Maschine-Kommunikation“), stellte sich uns im Januar 2014 als Bedrohungsszenario vor.

Google hatte den Thermostat- und Rauchmeldehersteller Nest Labs mit seinem „denkenden“ Thermostat Nest gekauft und in den Medien wurde daraufhin geunkt, dass es dem Internet-Riesen wohl vorrangig ums Datensammeln ginge. In diesem Fall um das Sammeln von Bewegungsdaten, die unsere Lebensgewohnheiten in den eigenen vier Wänden dokumentieren. $\mathrm{Zu}$ welchen Tages- und Nachtzeiten sind wir im Haus und welche Räume nutzen wir dann wie?

Zum einen sind diese Daten wichtig, um den Energieverbrauch daheim mit unserem Energieversorger zu koordinieren, sprich: die Heizung muss nicht durchlaufen, wenn keiner zu Hause ist. Zum anderen aber wird Google uns auf Grundlage dieser Datenerhebung fortan mit individualisierter Werbung versorgen, so viel sollte klar sein. Wie können diese Daten sonst noch genutzt werden? Werden Hacker sie vielleicht ausspionieren, um die Kommunikation der „Dinge im Internet“ zu manipulieren?

Andere Anwendungen machen den Zwiespalt zwischen hohem Alltagsnutzen durch intelligent miteinander agierende Werkzeuge im Netz einerseits und dem Anlegen undurchsichtiger Datenpools andererseits noch klarer: der Kühlschrank, der selbstständig die fehlenden Lebensmittel im Supermarkt nachbestellt, Autos, die untereinander Informationen über Staus austauschen, Herzschrittmacher, die im Notfall den Arzt alarmieren. Dies sind Projekte, die uns wie der mitden- 
kende Kühlschrank möglicherweise überflüssig erscheinen. Keiner wird jedoch abstreiten, dass umsichtig kommunizierende Herzschrittmacher oder PKWs, die uns an nervenden Staus vorbeilotsen, eine wirklich nützliche Innovation sind, die wir in der Regel dann auch haben wollen.

Das „Internet der Dinge“ ermöglicht industrielle Fertigungs- und Arbeitsprozesse vollkommen ohne menschlichen Anteil zu organisieren. Dass es sich dabei nicht um Science-Fiction handelt, sondern dies schon Realität in unserem Leben ist, zeigt die Zahl von 14 Milliarden Objekten, die heute bereits über das Internet vernetzt sind. Bis 2020 soll sich die Zahl nach Schätzungen des Marktforschungsunternehmens IDC (International Data Corporation) mehr als verdoppeln. ${ }^{1}$

Sprechen wir vom „Internet der Dinge“, so ist ein weiteres Trendwort nicht fern - „Smart“. Mit „Smart“ werden Technologien bezeichnet, die intelligent sind und sich effektiv bei der Bewältigung unseres Alltags einsetzen lassen.

Wir haben smarte Zahnbürsten, die uns sagen, wir sollten länger Zähne putzen, smarte Sensoren im Auto, die die Geschwindigkeit drosseln, wenn wir mal wieder im Straßenverkehr über die Stränge schlagen, und smarte digitale Fotorahmen, die sich ausschalten, wenn wir sie nicht mehr mit den Augen fixieren.

Und nicht zuletzt haben wir alle Smartphones, iPhones und iPads, die ohne unser Zutun einen sehr großen Teil unseres Alltags managen, indem sie diverse Tools und Services selbstständig miteinander vernetzen. Mobile Endgeräte werden zukünftig auch die zentralen Steuerungswerkzeuge in vernetzten Umgebungen sein.

Ohne Sensoren wie bei unserem denkenden Nest-Thermostat ist eine Vernetzung allerdings gar nicht möglich. Zunächst erfassen und analysieren sie Umgebungsdaten, bevor sie sie mit anderen Alltagsgegenständen austauschen. In Bibliotheken nutzen wir diese Sensoren längst in Form von RFID-Anwendungen.

Ergänzend können hier auch Projekte rund ums „intelligente Regal“ wie die des Sitterwerks in St. Gallen genannt werden, die es dem Bibliotheksnutzer mithilfe hochsensibler Sensoren ermöglichen, ein Medium in jeweils unterschiedliche inhaltliche Zusammenhänge zu stellen, ohne es aus den Augen zu verlieren und dabei Sachgruppen und Systematiken durcheinander $\mathrm{zu}$ bringen. ${ }^{2}$

$1 \mathrm{http://www.idc.com/getdoc.jsp?containerId=243661.}$

2 http://zukunftswerkstatt.wordpress.com/2013/03/12/zukunftsgestalter-in-bibliotheken-2013-2/. 


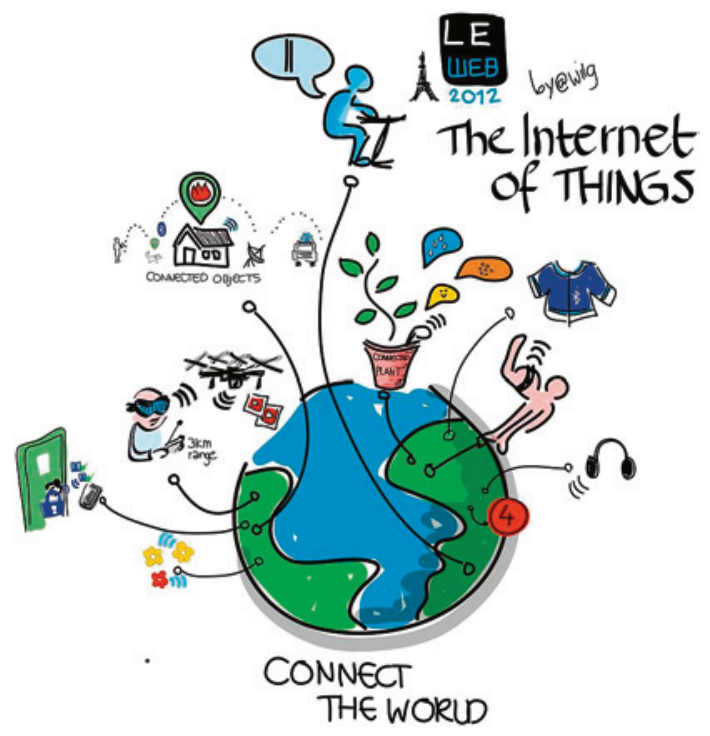

Abb. 1: Internet of things signed by the author CC BY 2.0; Wilgengebroed on Flickr https://www.flickr.com/photos/wilgengebroed/8249565455/.

Die rasante Weiterentwicklung dieser Sensoren und der wachsende Markt dafür

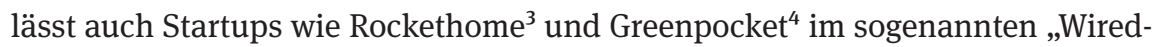
and-green-Markt“ aus dem Boden schießen, die mit Themen wie "Smart Home“ und „Smart Metering“5 den Weg in Richtung einer grünen Wohnzukunft ebnen. Zurzeit scheint eine flächendeckende Realisierung von Smart-Home-Konzepten jedoch noch zu aufwändig und zu teuer für die Wohnbaugesellschaften.

Wer sich einen Eindruck davon verschaffen will, kann sich z.B. auf der Website des „Museum of Science + Industry“ in Chicago über „Smart Homes“ mit smarter Technologie informieren. ${ }^{6}$ Es sind Best-Practice-Beispiele für das vernetzte Haus mit all seinen Vorteilen auch für die Umwelt.

3 http://www.rockethome.de/.

4 http://www.greenpocket.de/.

5 Smart Metering: Übertragungsvorgänge und die damit verbundenen Prozesse und Systemlösungen beim Einsatz intelligenter Zähler, die über die reine Energieverbrauchsmessung hinaus mit zusätzlichen Funktionen ausgestattet sind.

6 http://www.msichicago.org/whats-here/exhibits/smart-home/the-exhibit/green-inside-andout/smart-technology/. 
Abgesehen von vielen anderen „IoT“-Technologien, die hier leider zu kurz kommen müssen, wie die Tools der Quantified-Self-Bewegung ${ }^{7}$ - das Fuelband von Nike oder die Smart Watch „Samsung Galaxy Gear“ -, ist für mich die momentan bestechendste Vision des „Internets der Dinge“ die Verwirklichung eines umweltschonenderen Wohnens und Lebens in der Zukunft.

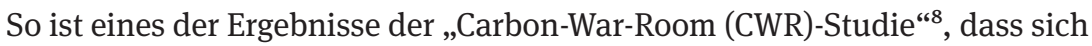
der CO2-Ausstoß auf der Erde um jährlich 9,1 Gigatonnen reduzieren ließe, wenn die zahllosen Maschinen, die wir nutzen, effektiver miteinander kommunizierten - ob nun im Haus, in der Landwirtschaft, in der industriellen Produktion oder im Verkehrs- und Gebäudemanagement.

Mit der Bewertung von Technologien des „Internets der Dinge“ bewegen wir uns, wie bei so vielen anderen Dingen auch, immer zwischen zwei Polen: Zum einen wollen wir unser Leben mit all seinen Aufgaben und Herausforderungen intelligenter, effektiver und ressourcenschonender, also insgesamt smarter organisieren. Und das nehmen uns die oben aufgeführten smarten Helferlein ja bereits ab. Damit sie das tun können, müssen wir allerdings Daten über unsere Lebensgewohnheiten preisgeben. Denn das eine wird ohne das andere nicht zu haben sein.

Wie ist das mit dem Datenschutz und in welchem Ausmaß werden die Maschinen bzw. „Dinge im Internet“ unser Verhalten möglicherweise unbemerkt normieren, wenn wir Menschen bei der „Maschine-zu-Maschine-Kommunikation“ außen vor bleiben?

Wir sollten daher innovative Technologien nicht nur auf ihren Nutzen hin testen und beurteilen, sondern immer auch abwägen, ob der individuelle Nutzen die Preisgabe der persönlichen Daten rechtfertigt. Im Rahmen der Vermittlung von Informations- und Medienkompetenz werden solche Fragen mit Sicherheit zukünftig auch Bibliotheksmitarbeiter beschäftigen.

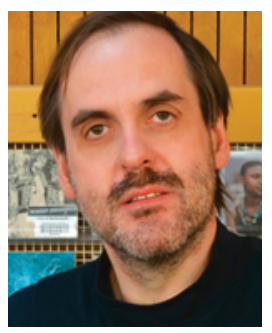

\section{Uwe Nüstedt}

Öffentlichkeitsarbeit in der Stadtbibliothek Wolfsburg

und Zukunftsentwickler bei der Zukunftswerkstatt

7 http://de.wikipedia.org/wiki/Quantified_Self.

$8 \mathrm{http} / / /$ www.grahampeacedesignmail.com/cwr/cwr_m2m_down_singles.pdf. 